\title{
Technique for Analyzing Relationship Between Unbalanced Load and Structural Deterioration in Outside Facilities
}

\author{
Soichi Ishikawa $a^{1, a,{ }^{*},}$ Gen Kobayashi ${ }^{1, b}$, Masaki Waki ${ }^{1, c}$, \\ Ryouichi Kaneko ${ }^{1, \mathrm{~d}}$, and Hiroaki Tanioka ${ }^{1, \mathrm{e}}$
}

${ }^{1}$ NTT Access Network Service Systems Laboratories, 1-7-1 Hanabatake Tsukuba, Ibaraki, Japan

asouichi.ishikawa.ck@hco.ntt.co.jp, bgen.kobayashi.wn@hco.ntt.co.jp, cmasaki.waki.me@hco.ntt.co.jp, dryouichi.kaneko.kv@hco.ntt.co.jp, ehiroaki.tanioka.nh@hco.ntt.co.jp

Keywords: Civil Structure, FEM, Utility Pole

\begin{abstract}
In order to provide telecommunication and FTTH services, NTT have installed so many facilities such as utility poles and optical cables. The number of poles is about 11.8 million and the total length of all installed cables is about 2.3 million $\mathrm{km}$. These facilities are now maintained and inspected visually by on-site maintenance staff at great expense. Therefore, we are researching a novel outside-facility renovation technology that increases cost-effectiveness by ensuring the long-term safe use of facilities. When a utility pole is newly constructed, it is designed under the assumption of a maximum load such as wind pressure being applied to the pole via cables and auxiliary attachments. However, the loads can become imbalanced when the guy wire cannot be tensioned as they cross private land or when there are obstacles, etc. We call this condition that unbalanced loads occur. Unbalanced loads are one of the key reasons for the structural deterioration of utility poles. After the deterioration occurs, the risk of pole collapse increases, because deflection and inclination are generated and finally the cracks propagate until collapse. The conventional solution is to replace the damaged pole. However, in many cases, the loads are created by other contiguous poles, so unbalanced loads occur again. Therefore, we are developing a method that can identify the reason by regarding sets of utility poles as "facility systems" and analyzing unbalanced loads quantitatively by FEM. In our research, simulation accuracy is improved by determining the quantitative differences obtained by comparing the calculated results obtained by FEM with measured values obtained by using a reaction wall. The simulations of structural degradation due to unbalanced loads taking into account the deformation of cables due to wind velocity and air temperature in addition to various material properties such as hysteresis and the sectional model of the utility poles. In addition to the above, the effect of soil is considered as a spring element, and the analysis involves the elements of horizontal spring (Kxi, Kzi) and vertical spring $(\mathrm{Kv})$. While the calculated and the measured values show good agreement, there is some small error. This error is considered to be due to the influence of parameters such as tensile and compressive strength, and we aim to improve the simulation accuracy by considering these effects in the future.
\end{abstract}

\section{Introduction}

We, NTT, have installed more than 11.8 million telephone poles (Here in after referred to as utility poles) and 2.28 million $\mathrm{km}$ of cable etc. in order to provide metallic and FTTH services such as telephone and Internet [1]. New construction, renewal and inspection work on these outside facilities are carried out at legally specified intervals, and their maintenance needs enormous time and labor costs. 
We have started using MMS (Mobile Mapping System) equipped with a laser scanner that can be driven on public roads, and 3D models of utility poles have been obtained from 3D point clouds. We estimate metrics of structural deterioration such as inclination and deflection, and put the data into practical use for inspection operations [2]- [4].

This study uses the mechanisms leading to structural deterioration to realize the long-term safe utilization of facilities.

In order to achieve this goal, we need two technologies. The one is "load visualization" which makes it possible to visualize where the unbalanced loads exist and their magnitude, which is a major factor triggering structural deterioration. Another is "the relationship between the unbalanced loads and the structural deterioration", which explains how to eliminate the unbalanced loads revealed by the visualization. This paper shows "the relationship between the unbalanced loads and the structural degradation".

\section{Load visualization technology and the relationship between the unbalanced load and structural deterioration}

When utility poles are newly constructed, the design assumes the maximum design load such as wind pressure and cable loading. However, unbalanced loads are likely to occur because guy wire cannot be constructed by the case of stringing on the private land and the existence of obstacles, etc. So, unbalanced loads are also generated even when cables are laid as designed.

A utility pole experiencing these unbalanced loads suffers deflection and inclination, after which cracking can occur that may trigger pole collapse. The 3D point clouds being gathered can measure the deflection and inclination with high-precision, and identify what needs to be inspected.

The purpose in this study is ensure that outside facilities can be used safely for longer periods than is now possible. The key is identifying unbalanced loads and their causes. This allows corrective measures to be undertaken which reduce renewal costs without sacrificing safety or security.

\section{Technology for understanding the relationship between unbalanced loads and structural deterioration}

Until now, structural deterioration has been judged by the visual inspection of every single utility pole. However, in the example shown in Fig.1, since the guy wire exists only at one end of a string of utility poles, the utility poles hosting the guy wire gradually incline due to the unbalanced loads. In this case, the defective utility pole identified by the conventional inspection regime (the second on the left) is renewed, but the fundamental problem causing the unbalanced loads remain. For that reason, the structural deterioration occurs again in a few years.

In order to solve the above problem, we introduce the concept that a set of utility poles is regarded as one "facility systems". As shown in Fig.2, when a defective utility pole is found, the entire system is inspected to clarify the cause of the unbalanced loads. Determining and rectifying the problem allows the outside facility to be safely utilized for longer periods than is currently available. Solutions to persistent unbalanced loads include the use poles of higher strength. 

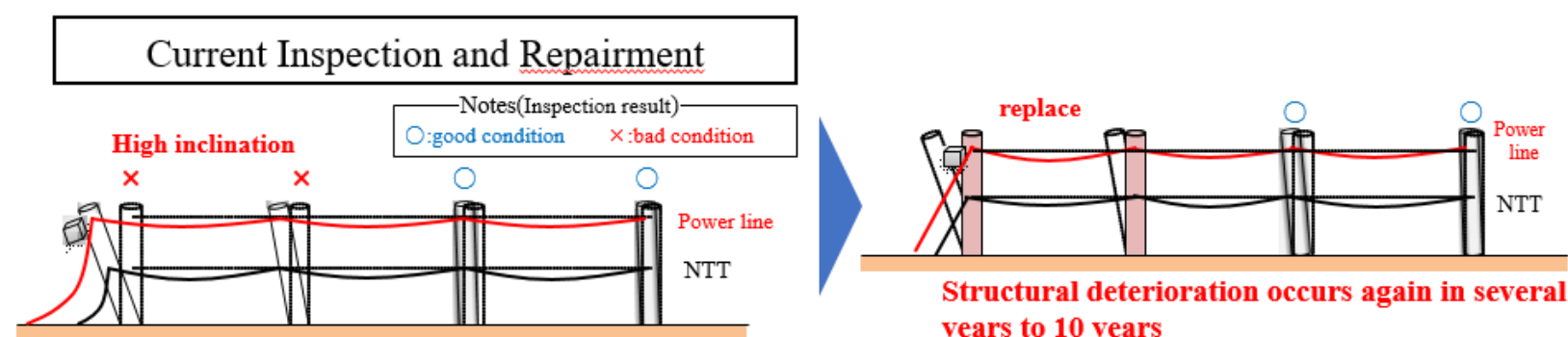
years to 10 years

Fig. 1: Examples of facility failures that cannot identified by the current inspection regime

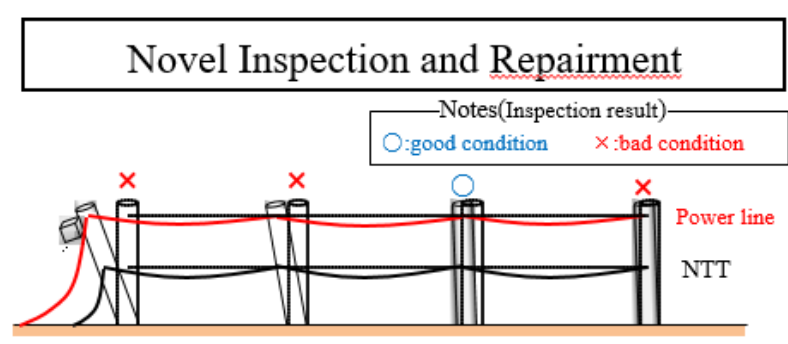

Fig.2: Examples of Renewal in "System" Repair guy wire
Prevent recurrence by installing brunch line

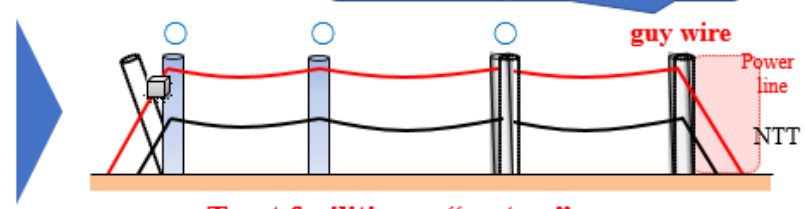

Treat facilities as "system", to solve root causes

For this examination, simulation accuracy is improved by comparing the calculated value using FEM (finite element method) with the measured value obtained from a load propagation experiment.

The simulation of the structural degradation due to unbalanced loads consider the deformation of cables due to wind pressure and air temperature, in addition to various material characteristics such as the cross-sectional model of utility poles and hysteresis as shown in Fig.3.
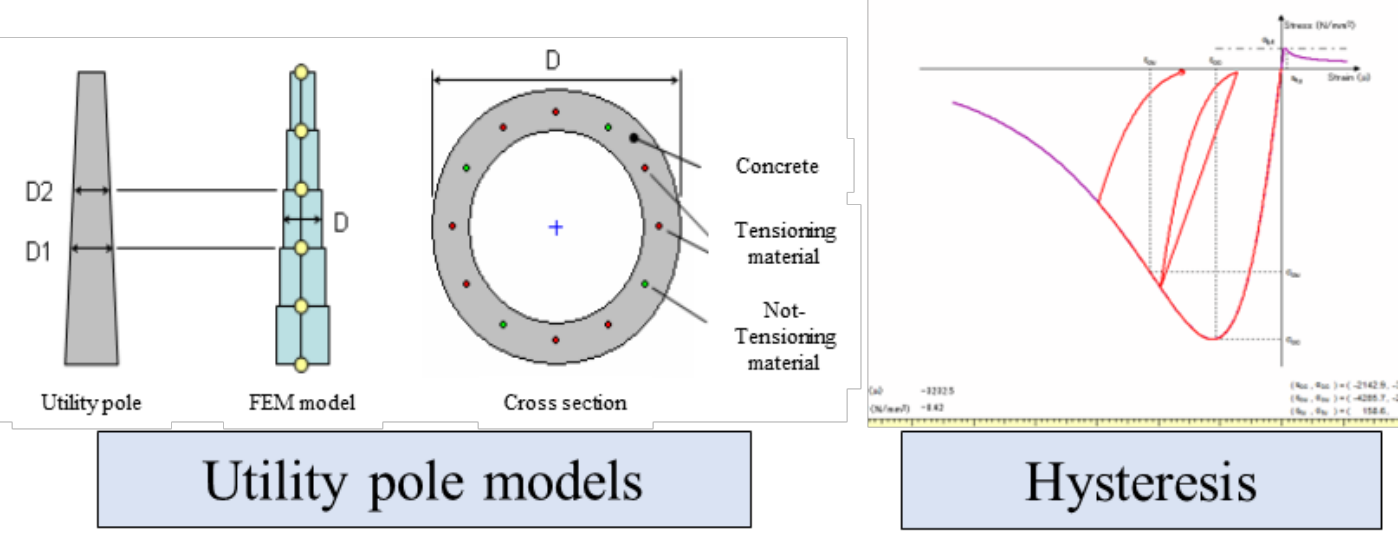

Fig.3: pole elements used in the simulation

When wind blows across the cable, the tension on the supporting utility poles increases, and the magnitude is given as follows.

$$
P_{c}=K \cdot \sum d \cdot S
$$


Here, $P_{c}$ represents the load in the cable, $K$ is coefficient that depends on the type of wind pressure load, $\sum d$ represents the sum of the outer diameters of the cable, and $S$ represents the average pole separation distance.

However, it is necessary to carry out the analysis three-dimensionally, because the wind direction is highly variable. In addition, if the ambient temperature is lower than when the cable was laid, the tension increases because the cable shrinks, while the opposite is true if the ambient temperature is higher. A unified expression is given below.

$$
d_{1}^{3}+\left\{\frac{3}{8} s^{2}\left(\frac{T_{0}}{E A}-\alpha\left(\theta_{1}-\theta_{0}\right)\right)-d_{0}^{2}\right\} d_{1}=\frac{3 W_{1} s^{4}}{64 E A}
$$

Variables are defined as $d_{0}$ : slack in no wind condition, $d_{1}$ : slack with wind, $S$ : distance between utility poles, $T_{0}$ : tension in no wind condition, $E$ : Young's modulus of cable, $A$ : cross-sectional area of cable, $\alpha$ : thermal expansion rate of cable, $\theta_{0}$ : temperature in installation condition, $\theta_{1}$ : current temperature, $W_{1}$ : cable weight per unit length are respectively expressed.

In addition to the above, the effect of soil reaction force is considered by assuming spring elements; the analysis considers the horizontal spring elements $\left(K_{x i}, K_{z i}\right)$ and vertical spring element $\left(K_{v}\right)$ as given by the following equation.

$$
\begin{aligned}
& K_{x i}=K_{z i}=K \cdot A_{h} \\
& K_{v}=K \cdot A_{v} \\
& A_{h}=D_{i} \cdot L_{i}
\end{aligned}
$$

Here, $K_{x i}, K_{z i}$ : horizontal ground spring elements, $K_{v}$ : vertical ground spring element, $K$ : ground reaction coefficient, $A_{h}$ : horizontal projected area of the pole member, and $A_{v}$ : area of the pole bottom face, respectively.

Fig. 4 shows the analytical results of the loads in a "facility systems" and the pole model before and after the analysis for a single utility pole. The figure shows that structural deterioration characteristics such as inclination and deflection occurred and unbalanced loads were generated in the left pole without guy wire as indicated by the FEM using equations (2) to (5) for pole models created using various material properties. This simulation of the system and a single body made it possible to elucidate the relationship between unbalanced loads and structural deterioration.

Next, we constructed an experimental setup capable of imposing loads on an actual utility pole. In order to accurately apply the load to the utility pole, a reaction wall, shown in Fig.5, was used.

Fig. 5 shows a birds-eye view of the reaction wall. The reaction wall can apply loads in excess of the largest design loads for the utility poles used by NTT. This system allows us to control the utility pole's deterioration, since the additional load could be accurately determined. In the future, the equipment which fixes the utility pole will be mounted so that the experiment except for the effect of the soil in "system" composed of multiple utility poles and cables may be possible. 

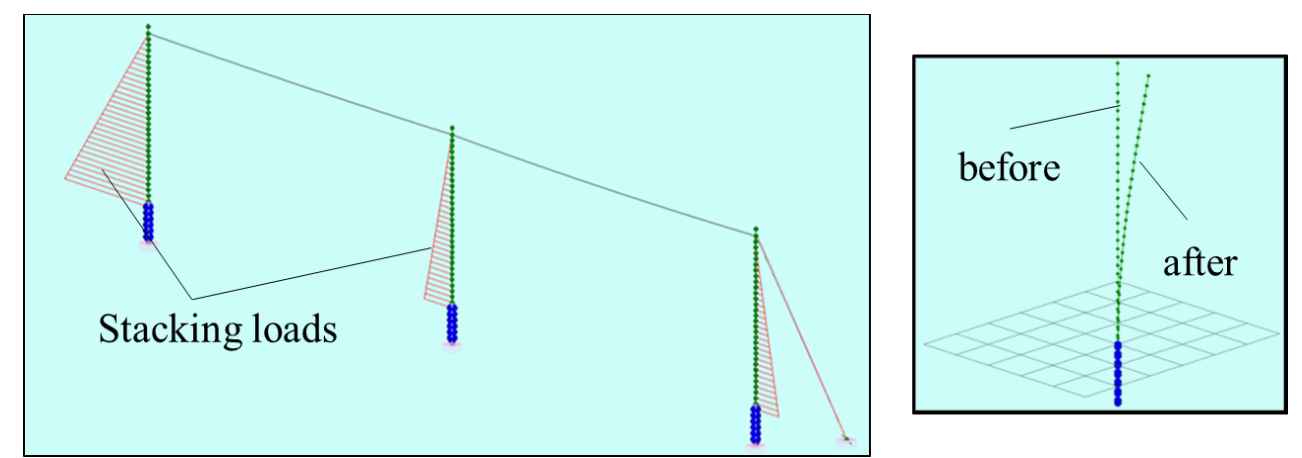

Fig4: Analysis of structural deterioration in utility poles using finite element method (FEM)
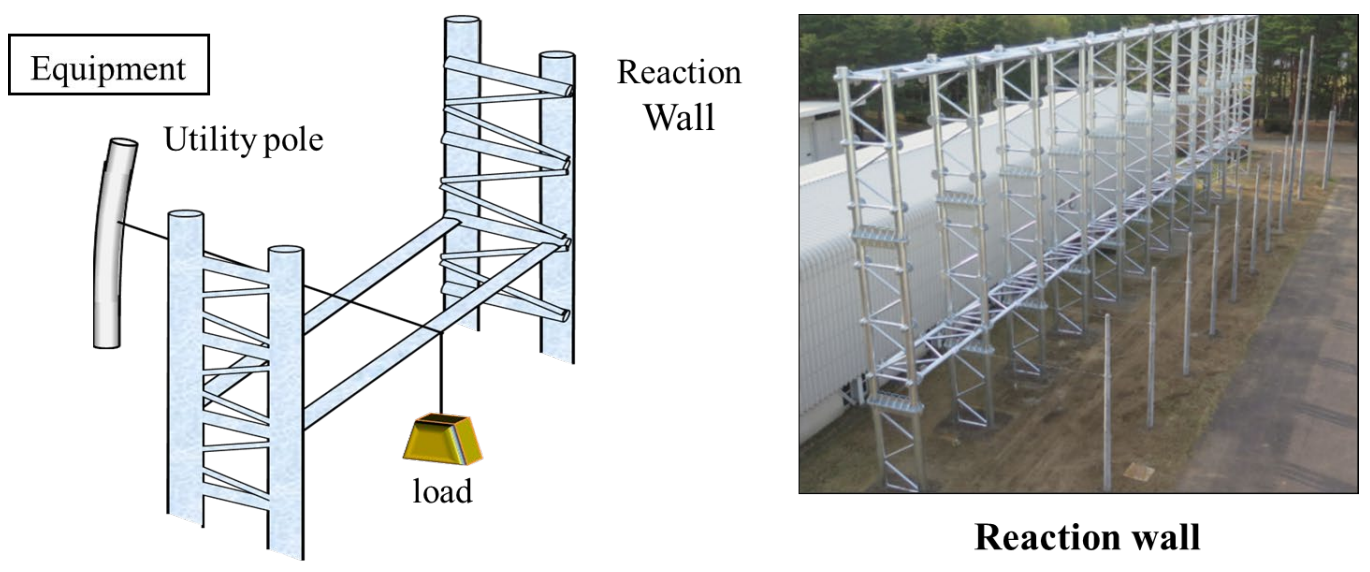

Fig5: Loading via Reaction Wall

Using the experimental setup shown in Fig.5, loading to $2 / 3^{\text {rd }}$ of the design load of the utility pole $(1.3 \mathrm{kN})$ was applied for up to 40 days and we measured deflection values. The results are compared with the simulation results in Fig.6. The measured deflection was captured by a fixed laser scanner. In this figure, the error between the calculated value and the measured value shows good agreement in the range of $2 \sigma$. However, since we are aiming to use this simulation for practical use, further improvement of the error to be within $1 \sigma$ is necessary. 


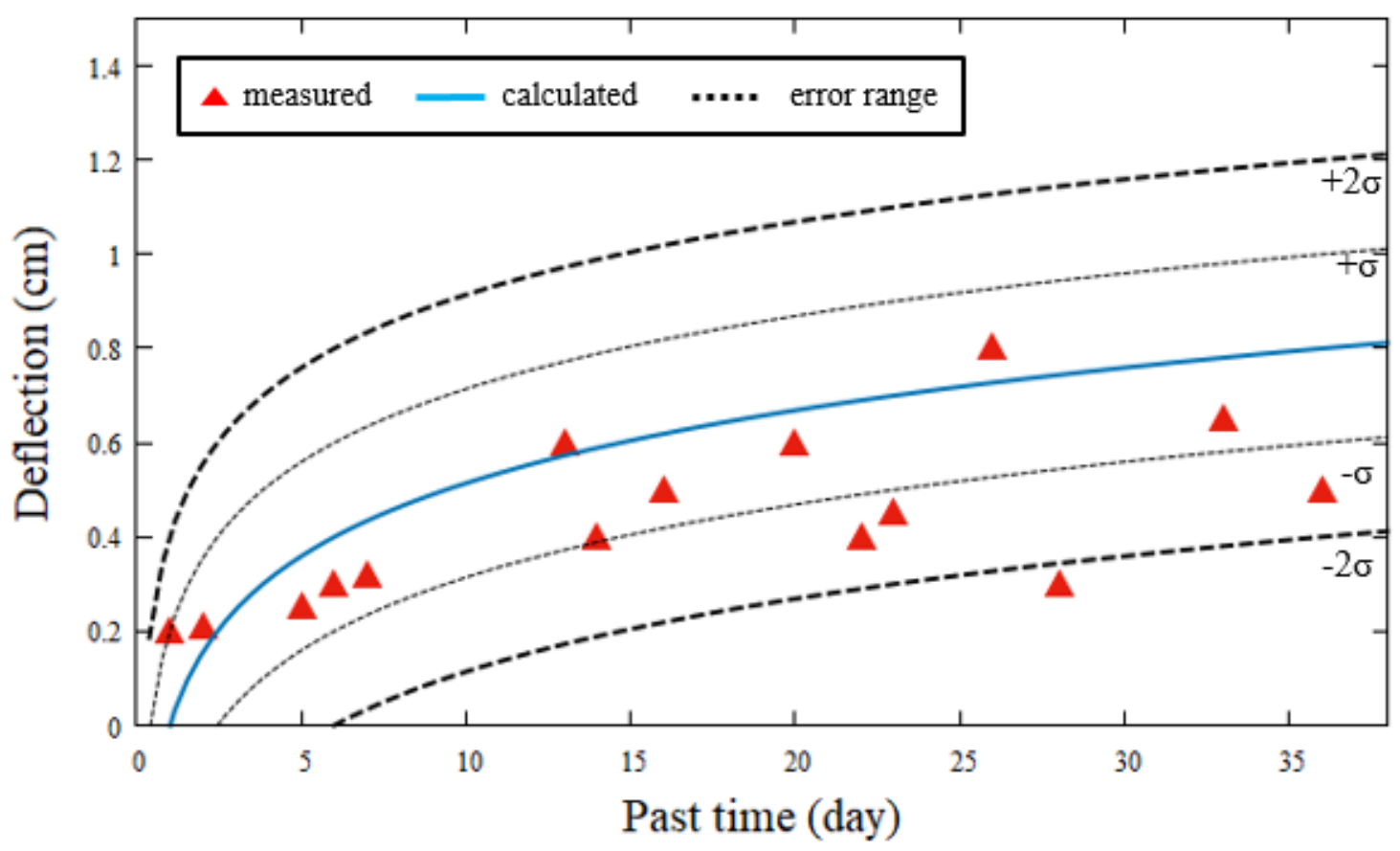

Fig6: Comparison of pole deflection due to loading over time

\section{Summary}

The paper detailed our proposal to identify the relationship between unbalanced loads and structural deterioration of utility poles. FEM-based simulations elucidated the structural deterioration with high precision. Research and development activities will continue to realize further enhancement of the proposal, and enable its application to outside facilities for practical use; the goal is to reduce the cost of maintenance activities without sacrificing safety or security.

\section{References}

[1] Information NTT East: Telecommunication Facilities, Information NTT East https://www.ntt-east.co.jp/databook/pdf/2019_allpage.pdf>, (Retrieved 27.7.2020)

[2] T. Kurashima, H. Ieda, and M. Waki: "Three-dimensional off-site access facility management technology using a mobile mapping system", 3rd Report, Shin-Gaku Gijutsuho, OFT2015-6, pp. 25-30, 2015.

[3] M. Waki, T. Goto, K. Katayama, and T. Kurashima: "A study for the application of three-dimensional facility management technology to maintenance and inspection work Three-dimensional off-site access facility management technology using mobile mapping system", 7th Report, Shin-Gaku Gijutsuho, OFT2016-22, pp. 25-28, 2016.

[4] M. Waki, T. Goto, and K. Katayama: "3D facility management technology using a mobile mapping system (MMS)", Communications Society Magazine of the Institute of Electronics, Information and Communication Engineers, vol. 12, no. 1, pp. 39-45, 2018. https://doi.org/10.1587/bplus.12.39 